\title{
Enhanced multiwavelength generation in Brillouin fiber laser with pump noise suppression technique
}

\begin{abstract}
A new multiwavelength Brillouin fiber laser (BFL) that provides a large number of Stokes lines with improved optical signal-to-noise ratio has been proposed and demonstrated. The BFL cavity is only formed by a nonlinear fiber loop mirror (NOLM) with $500 \mathrm{~m}$ long highly nonlinear fiber (HNLF). The BFL with improved performance is based on the suppression of the Brillouin pump noise floor utilizing a narrow tunable bandpass filter. The generation of Stokes lines covering up to a $33.67 \mathrm{~nm}$ wavelength range is achieved by setting the Brillouin pump signal within the HNLF's zero dispersion wavelength and with power of $250 \mathrm{~mW}$. This is owing to the combination of the stimulated Brillouin scattering and four-wave mixing effect in the NOLM structure.
\end{abstract}

Keyword: Multiwavelength generation; Brillouin fiber laser; Pump noise suppression technique 